\title{
Application of Ranging Difference Location Algorithm in Wireless Sensor Network Location
}

\author{
https://doi.org/10.3991/ijoe.v13i07.7285 \\ Bo Xiang \\ Chongqing Three Gorges Medical College, Chongqing, China \\ 56612659 @qq. com
}

\begin{abstract}
A kind of location technologies are developed used in the sensor network monitoring, the existing RSSI location algorithm are analyzed, and a localization method is proposed based on the SSDOA ranging difference method. hyperbolic equations are constructed and Chan's algorithm is used to solve the coordinates of unknown nodes. The simulation model of ranging error location algorithm is established by MATLAB. By analyzing the effect of ranging accuracy on positioning accuracy, the positioning performance of classical RSSI localization algorithm is compared. The results show that the WSN localization algorithm based on the SSDOA has a good localization performance and can meet the localization requirements of some WSN applications. Based on the above finding, It is concluded that Chan's algorithm is suitable for wireless sensor networks based on TDOA localization algorithm.
\end{abstract}

Keywords-SSDOA ranging difference method, positioning performance, wireless sensor network

\section{$1 \quad$ Introduction}

Wireless Sensor Network (WSN) is a new multidisciplinary research field in the world. It combines the sensor technology, embedded computing technology, MEMS technology, modern network, wireless communication technology and so on. It can be used for real-time monitoring, sensing and collecting information of all kinds of environment or monitoring objects through various integrated micro sensors. This information is transmitted wirelessly and transmitted to the user terminal in a selforganized and multi-hop network, thus realizing the connectivity of the physical world, the computing world and the ternary world of human society. Sensor network can make people at any time, place and environmental conditions to obtain a large number of detailed and reliable information. It has been widely used in military, national security, environmental monitoring, traffic management, medical and health, manufacturing and other fields [1-6].

After the wireless sensor network node in the network, the network began to enter the unknown node positioning. In this stage, the beacon nodes in the network need to broadcast the positioning data frame in turn. The unknown node measures the dis- 
tance from the received RSSI and determines its distance to the beacon node, while performing a positioning algorithm to find the unknown node coordinates.

In this paper, we propose a new approach based on SSDOA (Signals Strength Difference of Arrival). The localization mechanism first constructs the hyperbolic system of unknown nodes, and then uses the Chan's algorithm to estimate the unknown nodes. In general, Chan's algorithm is widely used in wireless sensor networks based on TDOA localization algorithm. It can take advantage of all the measured value of TDOA. Therefore, it is possible to reduce the influence of the individual large random errors on the positioning and obtain a better positioning effect.

\section{State of the Art}

RSSI positioning algorithm is based on the measured signal reception value, and then according to the formula to calculate the distance between the unknown node and the beacon node. The RSSI intensity value can be obtained either by an unknown node or by a beacon node. From the unknown node is called self-positioning, the latter is called telemetry positioning. In the self-localization mode, the target node can measure its distance from multiple beacon nodes in the network, and then calculate its position relative to the beacon node. The telemetry positioning is the opposite. The RSSI intensity of the unknown node is measured by the beacon node, and thus determines the location of the unknown node. For the self-localization mode, the radio transmission power of each beacon node is the same. For the telemetry positioning mode, the wireless sensor network system should have the power measurement function to determine the radio transmitting power of each unknown node, but also have the ability of data transmission [7-9]. The classical RSSI location algorithm is not high accuracy, the main reason is that the temperature, obstacle, propagation mode and so on in the experimental environment are often transform, especially the influence of multipath effect, makes the application of RSSI's localization algorithm in WSN difficult, and the localization performance of the algorithm is not satisfied.

\section{Methods}

The positioning scheme of RSSI is a method to estimate the position of unknown nodes by measuring the received radio frequency signal intensity. The key of this method is to estimate the distance between the unknown node and multiple beacon nodes by the attenuation degree of the signal, and then use these distances to estimate the coordinates of unknown nodes. In the practical application environment, the diffraction of radio waves is affected by the multipath propagation. The traditional RSSI method is not accurate.

In this paper, the SSDOA method obtains the distance estimation between the unknown node and the beacon node through RSSI ranging. The difference calculation for every two ranging values can form a hyperbolic equation. Therefore, a number of ranging values can form a bilinear system with respect to the coordinates of the un- 
known nodes, and the solution of the system can obtain the estimated position of unknown nodes.

Assuming that the coordinates of different beacon nodes are $\left(x_{i}, y_{i}\right)$, then the coordinate $(x, y)$ of the unknown node and the neighboring beacon nodes can determine different circles, that is,

$$
R_{i}=\sqrt{\left(x_{i}-x\right)^{2}+\left(y_{i}-y\right)^{2}}(i=1,2, \ldots M)
$$

Since the position of the beacon node is constant, the distance between any two beacon nodes is a constant value. For a planar positioning system, the unknown unknown node can be located on the hyperbola with the two beacon nodes as the focus, that is,

$$
R_{i, 1}=R_{i}-R_{1}=\sqrt{\left(x_{i}-x\right)^{2}+\left(y_{i}-y\right)^{2}}-\sqrt{\left(x_{1}-x\right)^{2}+\left(y_{1}-y\right)^{2}}
$$

In the equation $\mathrm{i}=2,3, \ldots \mathrm{M} ; \mathrm{M}$ is the number of beacon nodes in the network.

In SSDOA, the location performance depends on the accuracy of the path loss model, that is, the accuracy of the RSSI ranging phase. According to the signal attenuation, if the loss model can accurately estimate the signal transmission distance, then the positioning accuracy will be high; otherwise, the positioning accuracy will be poor. Therefore, the ranging technology used in this paper is based on RSSI ranging compensation in Chapter 3, which can maximize the accuracy of RSSI ranging and ensure the positioning performance of SSDOA.

According to a typical model of fading:

$$
d=10 \frac{R S S I\left(d_{0}\right)-R S S I(d)+\xi_{\sigma}}{10 \lambda} \times d_{0}
$$

The attenuation model of the RSSI experimental data was fitted:

$$
R S S I=-15.02 \ln (d)+315.78
$$

On the inverse function of the above formula and use the Taylor formula obtained:

$$
d \approx 0.321 \times R S S I^{2}+2.011 \times R S S I+53.22
$$

Therefore, by the formula (2) and formula (5) obtained the hyperbolic equations:

$$
\left\{\begin{array}{l}
R_{i, 1}=R_{i}-R_{1}=\sqrt{\left(x_{i}-x\right)^{2}+\left(y_{i}-y\right)^{2}}-\sqrt{\left(x_{1}-x\right)^{2}+\left(y_{1}-y\right)^{2}} \\
d \approx 0.321 \times R S S I^{2}+2.011 \times R S S I+53.22
\end{array}\right.
$$


In the equation $\mathrm{i}=2,3, \ldots \mathrm{M} ; \mathrm{M}$ is the number of wireless beacon nodes in the network. In combination with the ranging error compensation method, equation (6) becomes:

$$
\left\{\begin{array}{l}
R_{i, 1}=R_{i}-R_{1}=\sqrt{\left(x_{i}-x\right)^{2}+\left(y_{i}-y\right)^{2}}-\sqrt{\left(x_{1}-x\right)^{2}+\left(y_{1}-y\right)^{2}} \\
R_{j} \approx R_{j c}-\varphi(R S S I)
\end{array}\right.
$$

In the equation $\mathrm{i}=2,3, \ldots \mathrm{M} ; \mathrm{M}$ is the number of wireless beacon nodes in the network.

Multiple RSSI values can form a set of hyperbolic equations on the unknown nodes, the solution of the system can get the unknown coordinates of the location of the node.

Solving the system of equations (7) can obtain the coordinate position of the unknown node. At present, there are many methods for solving the linear equations, which have different requirements for the error of RSSI measurements, and the computational complexity is also different. Among them, Chan and Taylor are most commonly used.

The FANG algorithm can be used to obtain the analytical expression, but it can only use the measurement value equal to the number of unknown quantities, and cannot use more redundant information to improve the positioning accuracy. SI, SX can also be given analytic, but they get the suboptimal solution. Taylor series expansion method is a recursive algorithm that needs initial conditions. The algorithm is characterized by large computational complexity and can be applied to various channel environments. The accurate results can be obtained under the condition that the initial position is close to the actual position, but it is difficult to guarantee the convergence when the initial position is difficult to be determined. This also limits the accuracy of Taylor series expansion method in practical application.

Chan's algorithm is a non-recursive algorithm for solving hyperbolic equations. The algorithm uses two-step maximum likelihood estimation (MLE). Chan's algorithm not only has a small computational burden, but is easy to implement and has high localization accuracy under visible distance (LOS). Moreover, the Chan algorithm also takes advantage of all the RSSI values provided by the WSN network. It can greatly reduce the impact of individual large random measurement error, and get better positioning performance. Therefore, the hyperbolic model of Chan's algorithm is adopted as the mathematical model of the positioning system.

When an unknown node gets the RSSI of multiple beacon nodes, a set of hyperbolic equations about the unknown node is obtained from the hyperbolic model (2). On the two sides of the equation to simplify the square, so that $K_{i}=x_{i}^{2}+y_{i}^{2}$, the following equations can be obtained:

$$
R_{i, 1}^{2}+2 R_{i, 1}=K_{i}-2 X_{i, 1} y-K_{1}, i=2,3, \ldots M
$$


In the equation $X_{i, 1}=x_{i}-x_{1}, Y_{i, 1}=y_{i}-y_{1}$; In summary, the calculated position coordinates of the improved unknown point are:

$$
z_{c}^{\prime} \approx\left(G_{c}^{T} B^{-1} G_{c}^{T} Q^{-1} G_{c} B^{\prime-1} G_{c}^{\prime}\right)^{-1} \times\left(G_{c}^{T} B^{-1} G_{c}^{T} Q^{-1} G_{c} B^{\prime-1}\right) h^{\prime}
$$

Among them,

$$
\begin{gathered}
z_{c}^{\prime}=\left[\begin{array}{l}
\left(x-x_{1}\right)^{2} \\
\left(y-y_{1}\right)^{2}
\end{array}\right], \quad B^{\prime}=\left[\begin{array}{ccc}
z_{c}(1)-x_{1} & 0 & 0 \\
0 & z_{c}(2)-y_{1} & 0 \\
0 & 0 & z_{c}(3)
\end{array}\right], \\
h^{\prime}=\left[\begin{array}{l}
\left(Z_{c, 1}-x_{1}\right)^{2} \\
\left(Z_{c, 2}-y_{1}\right)^{2} \\
Z_{c, 3}{ }^{2}
\end{array}\right], G_{c}^{\prime}=\left[\begin{array}{ll}
1 & 0 \\
0 & 1 \\
1 & 1
\end{array}\right]
\end{gathered}
$$

[1] Then, the position coordinate calculation result of the final unknown node is:

$$
z_{p}= \pm z_{c}+\left[\begin{array}{l}
x_{1} \\
y_{1}
\end{array}\right]
$$

In practical applications, the RSSI value is often because of the environment, the test node difference and other factors have a lot of uncertainty, resulting in a larger positioning error. Therefore, this paper adopts the method of optimizing the selection of beacon nodes to improve the accuracy of nodes to be located. Due to the different location of the nodes, the network topology, connectivity and interference types are different. In each positioning performed in practice, $\mathrm{M}$ numbered beacon nodes are set in the monitored area environment, and the transmission power (fifth power) of the beacon node is unified.

M beacon nodes can obtain their own positioning coordinates by GPS or manual arrangement, and each node in the network has an address information record node. The bigger the RSSI value is, the closer the beacon node is to the unknown node, the smaller the error of ranging error. So, when the unknown node is located, the beacon node with the strongest RSSI value is selected from all the beacon nodes that can communicate with the unknown node, the RSSI value of the beacon node is read and the address of the beacon node is recorded. Combined with the above-mentioned localization algorithm model, the position of the unknown node can be well estimated.

Based on the above discussion, this paper presents the process of the optimal beacon node localization algorithm as follows:

In the WSN network, a certain number of beacon nodes are set in advance, and it is assumed that the position coordinates of each beacon node are known and the address of the record itself is assigned to each beacon node, and an address is provided for the preferred beacon node. 
When the WSN starts to locate the unknown node, in order to transmit all the unknown nodes, the beacon node sequentially transmits the broadcast positioning packet to each unknown node and reads the RSSI value, and the RSSI values are sorted for all beacon nodes.

Select the bearer node with the highest RSSI value as the reference node, and read the address of the beacon node to locate the selected beacon node, and take out the corresponding RSSI value.

The distance between the best beacon node and the unknown node is estimated for the selected RSSI, and the error of the estimated distance is compensated to improve the accuracy of the estimated value.

The corresponding RSSI difference positioning model is established, and the Chars algorithm is used to solve the difference positioning model. Then, the position estimation coordinates of unknown nodes are obtained.

For simulation, the simulation environment is a $10 \times 10 \mathrm{~m}^{2}$ square area. 30 unknown nodes distributed in the square area, a beacon node is arranged at each of the four corners of the square.

\section{$4 \quad$ Experiment and Results}

Due to the limitation of experimental conditions, it is difficult to carry out the localization experiments on a large number of nodes. Although the calculation of Chan algorithm is small, for the LPC 2138 is still a very computationally intensive work. At the same time, LPC2138 after a large number of multiplication and division operations, the calculation accuracy will be errors. In view of the above considerations, this paper uses Matlab7.0 to simulate the difference location algorithm of RSSI ranging to further study the positioning accuracy of the algorithm. The positioning algorithm in the network protocol for the application layer, so in order to simplify the simulation program, the simulation does not involve the physical layer, the MAC layer and the network layer of the node, but according to the actual needs of positioning, mainly for the application layer positioning error and the unknown node to the beacon node distance measurement error to explore.

In Figure 1, when the distance measurement error increases by $1 \%$, the positioning error of the algorithm is increased. Among them, " $\times$ " represents the average positioning error of 30 unknown nodes in the above simulation, "+" represents the minimum error of positioning error in 30 unknown nodes, "-" represents the maximum value of the positioning error in the unknown node. When the average positioning error reaches $50 \%$, the average positioning error of the 30 unknown nodes is close to 20 , and the maximum positioning error reflects the upper and lower limits of the node positioning error. It can be seen that the localization accuracy of the ranging error localization algorithm can be effectively improved under the premise of effectively improving the ranging accuracy of the node and reducing the ranging error.

Set $10 \times 10 \mathrm{~m}^{2}$ square area, 30 unknown nodes distributed in the square area, a beacon node is arranged at each of the four corners of the square. When the positioning phase starts to enter the ranging and positioning stage, the beacon node broadcasts the 
positioning data frame in sequence, and the unknown node is positioned in the square area. The simulation program locates using classical RSSI positioning algorithm and ranging difference location algorithm. The classic RSSI positioning method is shown in Figure 2.

The comparison between the classical RSSI location algorithm and the average positioning error of the ranging difference location algorithm is as shown in Figure 3.

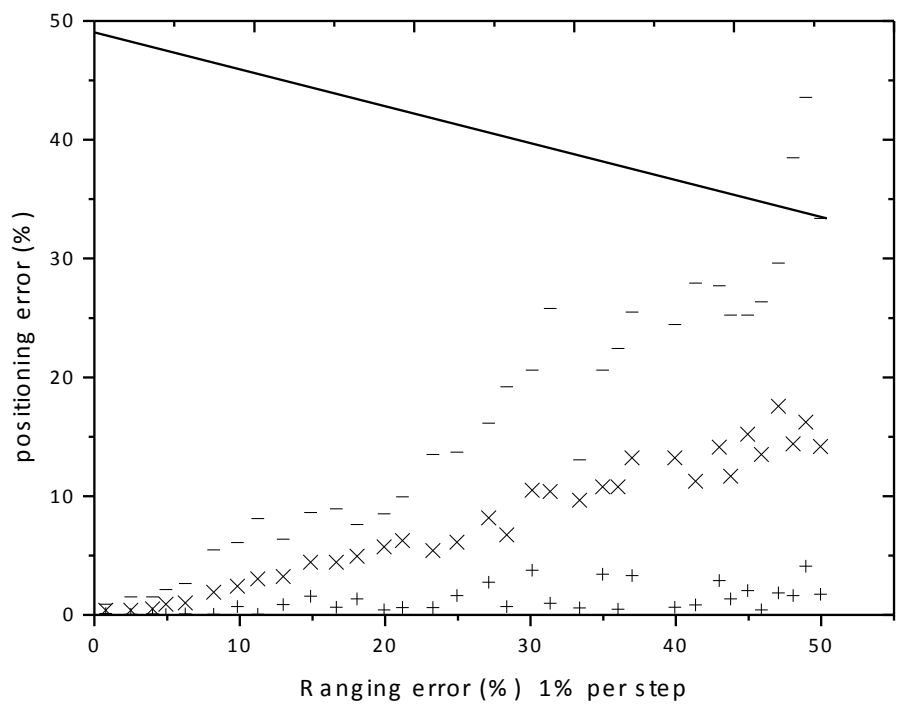

Fig. 1. Positioning error - ranging error

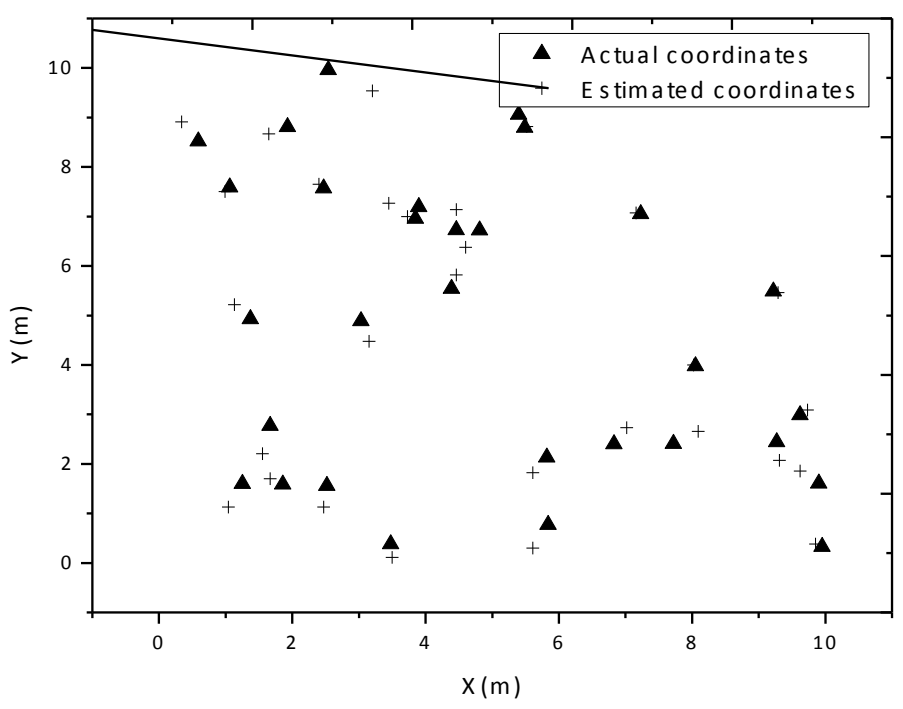

Fig. 2. Classic RSSI positioning 


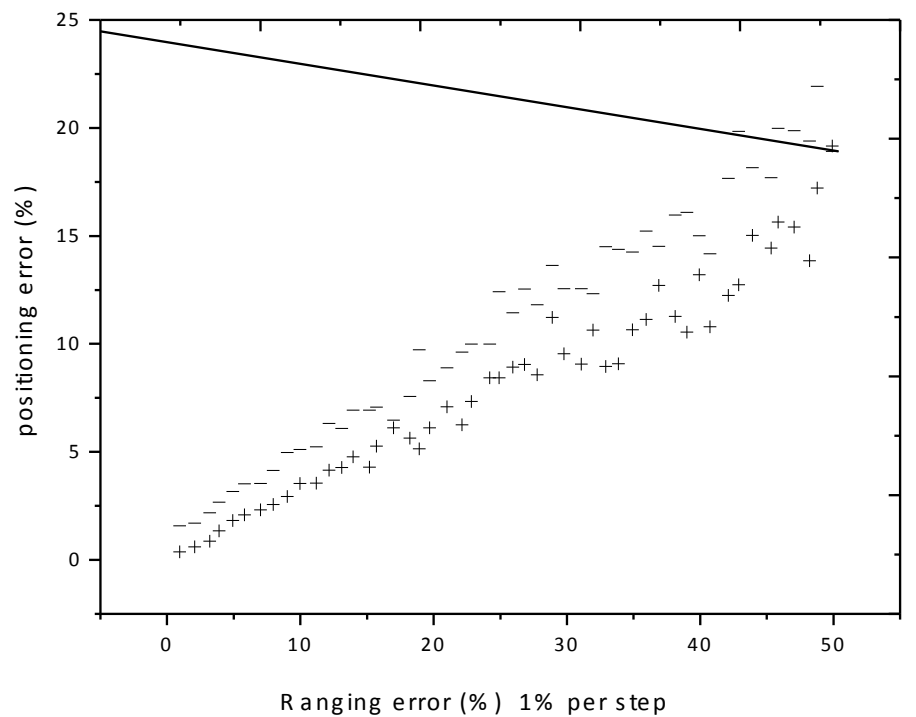

Fig. 3. Positioning error comparison chart

As the error trend shown in Figure 3, "-" represents the average positioning error percentage of the classical RSSI localization algorithm, "+" represents the average positioning error percentage of the ranging difference positioning algorithm. When the ranging error increases by $1 \%$, the average location error of the two localization algorithms will increases with the increase of the ranging error. When the ranging error is less than $12 \%$, the average positioning error of the two localization algorithms is in a stuck state. However, the average positioning error of the classical RSSI positioning algorithm is still high, that is, the positioning error of the ranging algorithm is slightly better. When the ranging error is greater than $12 \%$, the average localization error trend of the two localization algorithms begins to separate. The average localization error of the classical localization algorithm is higher than the average positioning error value of the ranging difference localization algorithm. At this time, the localization performance of the ranging difference location algorithm is better than that of the classical algorithm. Therefore, compared with the positioning performance of the two, the positioning error of the positioning algorithm is superior.

To sum up, for the SSDOA location difference algorithm proposed in this paper, two positioning simulations are carried out before and after the ranging error compensation. The results show that the improvement of ranging accuracy can ensure the localization accuracy of the algorithm, and improve the localization performance of the algorithm. On the contrary, it affects the localization performance of the algorithm. At the same time, the simulation results show that the location algorithm of distance measurement is better than the classical RSSI algorithm. Therefore, the proposed algorithm can be applied to some sensor networks. 


\section{Conclusion}

The positioning mechanism of wireless sensor networks based on RSSI location algorithm is proposed in this paper. In addition, the classical RSSI location algorithm and RSSI fading model are analyzed. Based on the analysis of the existing RSSI positioning algorithm, we put forward the positioning idea of the ranging difference method, construct the hyperbolic algorithm model. The Chan algorithm is used to solve the coordinates of unknown nodes under the precondition of determining the optimal beacon. By comparison and simulation, the results show that the WSN localization algorithm based on the SSDOA has a good localization performance and can meet the localization requirements of some WSN applications. From the experiment results, we draw some conclusions. Firstly, the effectiveness of the new RSSI algorithm is verified by simulation, and it is found that the positioning accuracy, positioning time and computational complexity have been improved significantly. Secondly, in the RSSI localization algorithm, an anchor node selection mechanism is added. That is, the best anchor node in the communication radius is selected to reduce the accumulation of distance error, and further improve the positioning accuracy.

\section{References}

[2] Ferdoush, S. and Li, X. (2014). Wireless sensor network system design using Raspberry Pi and Arduino for environmental monitoring applications. Procedia Computer Science, 34: 103-110. https://doi.org/10.1016/j.procs.2014.07.059

[3] Gutiérrez, J., Villa-Medina, J.F., Nieto-Garibay, A. and Porta-Gándara, M.Á. (2014). Automated irrigation system using a wireless sensor network and GPRS module. IEEE transactions on instrumentation and measurement, 63(1): 166-176. https://doi.org/10.1109/TIM. 2013.2276487

[4] Kajioka, S., Mori, T., Uchiya, T., Takumi, I. and Matsuo, H. (2014). October. Experiment of indoor position presumption based on RSSI of Bluetooth LE beacon. In Consumer Electronics (GCCE), 2014 IEEE 3rd Global Conference on (pp. 337-339). IEEE.

[5] Tuna, G., Gungor, V.C. and Gulez, K. (2014). An autonomous wireless sensor network deployment system using mobile robots for human existence detection in case of disasters. Ad Hoc Networks, 13: 54-68. https://doi.org/10.1016/j.adhoc.2012.06.006

[6] Jing, H.C. (2014).Coverage holes recovery algorithm based on nodes balance distance of underwater wireless sensor network. International Journal on Smart Sensing and Intelligent Systems, 7(4):1890-1907.

[7] Visser, H.J. and Vullers, R.J. (2013). RF energy harvesting and transport for wireless sensor network applications: Principles and requirements. Proceedings of the IEEE, 101(6): 1410-1423. https://doi.org/10.1109/JPROC.2013.2250891

[8] Wang, Y., Yang, X., Zhao, Y., Liu, Y. and Cuthbert, L. (2013). January. Bluetooth positioning using RSSI and triangulation methods. In Consumer Communications and Networking Conference (CCNC), 2013 IEEE (pp. 837-842). IEEE.

[9] Xie, S. and Wang, Y. (2014). Construction of tree network with limited delivery latency in homogeneous wireless sensor networks. Wireless personal communications, 78(1): 231246. https://doi.org/10.1007/s11277-014-1748-5 
Paper-Application of Ranging Difference Location Algorithm in Wireless Sensor Network Location

[10] Yang, Z., Zhou, Z. and Liu, Y. (2013). From RSSI to CSI: Indoor localization via channel response. ACM Computing Surveys (CSUR), 46(2): 1-32. https://doi.org/10.1145/254 $\underline{3581.2543592}$

\section{Author}

Bo Xiang is with the Department of Public Foundation, Chongqing three Gorges Medical College, Chongqing, China(56612659@qq.com).

Article submitted 13 June 2017. Published as resubmitted by the author 15 July 2017. 